\title{
Emerging themes and recommendations
}

Editorial team

Actions often ripple far beyond their immediate objective, and remembering this is reason to live by principle and act in hope that what you do matters, even when results are unlikely to be immediate or obvious.

(Solnit, 2017)

In the Introduction we asked you to imagine working and studying and researching and learning in a culture where "mutuality is the ethos shaping our interaction [...] where we can all be who we are [...] living the truth that we are all 'created equal'” (bell hooks, 2000, p.x).

As the recipes and articles throughout the book demonstrate, there are places within the University of Edinburgh which have encouraged and enabled systemic cultural change with the aim of embedding gender equality in practice. In Lumby's (2009) terms, this is when the institution is working with diversity, not just for (or towards) diversity; and when people are internally motivated to challenge and change biases in themselves as well as in others and in the system (Carnes et al., 2012).

Based on reflection about our authors' contributions, and our experience and learning during the project, we present recommendations which we hope will help University leaders, staff and students shape a principled and equitable future for all.

\section{What have our authors told us?}

We examined the recipes and papers through two main lenses: purpose and meta-theme. We asked what 
the authors' underlying purposes were in writing their recipes and articles. As referenced in the Introduction, we found content that was:

- $\quad$ Practical: documenting steps which worked for real change on an institutional level (14); on a personal level (8) and across a group of people (4).

- Informative: exploring a topic in depth (23); introducing a topic (4) and providing illuminating facts (5).

- Illuminating: describing authors' pivotal moments (4), and other content that reveals what it feels like to be the author - giving an insight into other lives (27).

We looked for recurring themes across the different types and focuses of content and found the following:

- Responsibility: of individuals, groups, departments and the University to identify, challenge and change inequalities and biases wherever and whenever they are encountered.

- Reflection: as each author considered what they are documenting, and how and what their own contribution might have been to both the problem and the response.

- Challenging bias and denial (own and others'): a natural follow-on from reflection. We noted feelings expressed and honoured; courage; and emotional labour (paid) and emotional work (unpaid).

- Language: the importance of language - what is said, how it is said, and what is actually meant, and what is heard. The power of the 'papercut comment' to undermine and diminish - whether meant or not - should never be underestimated.

- Well-being: this theme was implicit in the central thesis that if it is better for women then it is better for everyone, but emerged unexpectedly strongly across the different contributions as a desirable state worth striving for.

- Humour: often the recipes are funny - wryly, and ironically - giving a strong sense of the authors' voices. The humour in some of the proposed responses and solutions to difficulties makes the message easier to put across.

- Personal skills: the list of personal skills grew as we looked at the recipes in particular. Listening, appreciation, empathy, curiosity and imagination were especially noteworthy.

- Personal principles: congruence, integrity, sincerity, and again courage figured in many of the recipes, especially where the authors had had to make some kind of stand about poor, but long-accepted, practice.

- Processes: overlapping with the practical purposes above, many of the recipes described how the process of reflecting, identifying, changing, and sustaining that change was in and of itself as important as the change that was made.

The writers document how they, along with their colleagues and students, are quietly and firmly dismantling the stereotypes and inequities that have for so long got in the way of, as our initial statement affirmed, "embracing differences to create a more vibrant and rigorous intellectual, supportive and learning context for all our community". In doing so, they give us all practical guidance, ideas and a route map towards gender equality.

A colleague once exclaimed in exasperation at yet another organisational Grand Plan: "Milestones! I'm sick of milestones. I want inch pebbles!" The EqualBITE project in itself is a significant milestone on the University of Edinburgh's journey towards greater equality; this article sums up the inch pebbles that the authors - all of them - recommend. 


\section{Change for an equitable future}

The first step is to recognise that you have a problem.

The above quotation and others at the start of each subsection are by senior leaders in the University of Edinburgh (see Leadership perspectives on gender equality).

The model of change that we proposed at the start of this book notes that changes are driven from the inside out, and from the outside in: individual changes drive team changes, which in turn make shifts at a departmental and school level. And at the same time, societal changes drive policy changes which impact the institution and require change from institutional to individual level. The introduction of Athena SWAN has driven much of this change, but would not have worked had there not been substantial societal change to make it more than just a 'nice-to-have' tick-box exercise.

There is a Scottish phrase "It's aye been" which means things have always been that way, always will be that way, that's just how things are, and don't start rocking the boat now. This can be the most difficult aspect of culture to shift, especially in an institution with ancient and honourable traditions, but where tradition for its own sake can reinforce the blocks to healthy and necessary change. The authors recognise and challenge this: Leadership styles and approaches in Geosciences recognises different professional and leadership styles - not just 'alpha male' research star professor; The power of language: moving beyond past harms and present hurts looks at how language can either reinforce or shift barriers; and Women, competition and beliefs and Stereotype threat explore external and personal/internal obstructions to change.

Once gender inequalities are seen, then they cannot - and should not - be unseen.

\section{Our recommendations for action}

EqualBITE is pragmatic as well as visionary. We propose a set of practical recommendations emerging from the meta-themes and the recipe purposes; taken together these recommendations are what our authors have found can create sustainable culture change in practice.

1. Close the gap between policy and practice.

2. Take a pragmatic, evidence-based approach to implementing equity.

3. Involve everyone to attain equity.

4. Insist upon and model open and honest awareness, reflection and action.

5. Know, develop and celebrate yourself.

6. Remove bias (conscious and unconscious) in learning and teaching.

7. Continuously balancing gender is the outcome.

8. Create an environment that enables people to flourish .

9. Recognise and honour the greater context.

10. Be a beacon.

\section{Close the gap between} policy and practice

If you want something to be adopted, you have to do it and pay attention to it. People look to what you do, not what you say.

People believe what is done, not what is said. How values are implemented in practice really matters. Most people will say that being equal or fair is a good thing - this is a high-level value and few would disagree with it. But is there congruence between what you say you value, and what you do?

Which workplace policies and behaviours do you say are valuable and which do you reward? Much of this book documents the authors' experiences of messy bits between policy and action; for example, the recipe Flexible working: being realistic looks at what can happen when flexible hours and workload expectations do not match. This is a problem highlighted not just in the 
recipe, but also in Leadership perspectives on gender equality, and in Gender in higher education: the current landscape in the UK. Without congruence there can be a double problem - not only will some issues not be addressed, they will be actively suppressed because people believe that there is a system in place to prevent them.

Universities need to live up to their stated values if they want to earn and keep their students' respect. The two contributions about anti-harassment campaigns one from a student representative, and one from the University - illustrate the importance of this (see A reflection on EUSA sexual harassment campaigns and $A$ reflection on the University of Edinburgh's policy on sexual harassment).

The meta-themes crucial to doing what we say we do, are: responsibility of individuals, groups, departments and the institution to maintain and practise values-driven behaviours day by day; language - being aware of whether what is said, and what is meant, and what is heard are congruent and trustworthy; and maintaining our individual personal principles of integrity, sincerity and courage to stand up for what is valued regardless of pressure.

Linking the operational to the aims of an organisation requires significant effort and ongoing maintenance. Importantly, it requires a continuous process of review, reflection, and adjustment, paying attention to process as well as outcomes, to be enacted at all stages of aims, strategy, policy and operational design; regularly, efficiently and effectively. The Athena SWAN process of award renewal is a useful starting point.

There are recipes and content clusters that demonstrate excellent practice, and others that show what happens, and how to respond, when there is a mismatch between espoused values and actual practice. Among the many recipes and articles are: Flexible working - being realistic, Gender balancing your seminar speakers, Advertise all opportunities large and small,
What have you done to my squash courts!, How to run more equal meetings, How to become a better scientific evaluator.

\section{Take a pragmatic, evidence-based approach to implementing equity}

You need a baseline to track progress. Athena SWAN is helpful with this.

Nurturing and growing gender equality through a pragmatic evidence-based approach requires as much rigour as any other piece of research. Collect good data and regularly assess what is unknown. Where are the gaps? What might we not be seeing because of our own unconscious bias? Any enquiry into gender equity has to start with values, not data. What does the organisation and culture value? How might this value be measured? If it is done the other way around, data will drive culture and this will introduce unintended biases and assumptions. Data should be a way of checking and informing; not a way of driving and directing organisations; this is, perhaps, one of the key benefits of Athena SWAN.

There is much good research already out there that can be used to inform policy and processes - not just the data the organisation collects but the body of knowledge in other fields. We have, for example, used evidence-based research to inform our thinking in developing A model for change, in particular research on gender bias as a remediable habit (Carnes et al., 2012), and the review of evidence from a behavioural economics perspective (Bohnet, 2016).

Many of the required data sets relating to gender equality are now publicly available. This transparency makes the task of monitoring the impact of change easier. However, there are still areas which lack transparency. For example, the Guardian newspaper's report on sexual harassment in UK universities between 2011/12 and March 2017, characterised the level of staffstudent harassment as an "epidemic". 
The article was based on data from freedom of information requests to universities within the UK. Rachel Krys, co-director of the End Violence Against Women Coalition, quoted in the Guardian article said:

We know that institutions which take tackling sexual harassment and violence seriously, and have policies and systems in place to encourage reporting and train staff to deal with complaints as they arise, report higher numbers. It's the universities which aren't dealing with this openly which perversely appear to have fewer problems. (Batty, et al., 2017)

The report raises a potentially troubling issue but it is difficult to accurately gauge the nature and the extent of the problem without more robust data and careful analysis. While these are delicate issues, universities do employ researchers with the requisite expertise to sensitively gather and sift the evidence.

Meta-themes here are responsibility, reflection, personal principles, and processes. Responsibility for determining and maintaining a good process for ongoing checking of data-informed decisions; processes for reporting data analysis conditions and contingencies as clearly as the results themselves (the conditions under which some data are valid, or not, matter just as much as those data themselves); reflection on possible biases; and principle in clearly communicating how decisions made relate to the data. Relevant recipes and papers include: Sport and exercise for all, Challenging bias, $A$ model for change, Gender and the Research Excellence Framework, Defining excellence.

\section{Involve everyone to attain equity}

Championing a wide range of role models is important.

Everyone can and should benefit from a more equitable working environment and culture. To achieve this, everyone has to contribute to its success. Without exception.

Carnes et al. (2012) identify the moment of real change in addressing and amending habits of gender bias when motivation switches from external to internal drivers. They observed people moving from "I don't want to appear prejudiced to my colleagues" to a realisation that "I can do it" and "I will benefit from doing it" as the advantages of rectifying bias became apparent. This is known informally as the WIIFM factor - What's In It For Me - and reframes old attitudes toward equality work ('making up', 'redressing') as 'great improvements'.

This can be a huge challenge when the wider, changing social and political context is considered. Athena SWAN, as Judy Robertson has observed in her paper The current landscape at the University of Edinburgh can sometimes be perceived as a duty rather than a wholeheartedly embraced opportunity for change. Our authors suggest that we should pay attention to our processes - how we recruit, interview, and take on staff and students; what language we use to do this; how we build diversity within groups, rather than between them. Be aware of critical mass for example rather than distributing a small number of female students between groups so they end up as the only woman, make sure that one-third of students in a group are female, even if that means some maleonly groups. Recipes and papers direct us to lead by example, supporting each other when we challenge inequitable behaviour and language, and demonstrating its opposite. This means being aware of one's own privilege - it is not the same as entitlement. With privilege comes a significant responsibility; acknowledge it and check it as the language of privilege can be unhelpful, even damaging.

Many of the recipes document steps that have worked for real personal and group change, pivotal moments, and a sense of what it is like to step into someone else's shoes. The meta-themes of personal 
principles and personal skills talk of courage: to refuse to accept 'bear-pit' behaviour, and to challenge it when it rears its ugly head.

One senior woman leader said: "There is a risk when you do call out bad behaviour there is a backlash. I wouldn't be able to call out bad behaviour until I got to this level of seniority. And now I am at this level I feel I have a responsibility to". Another senior woman, talking about 'the bear-pit' in an EqualBITE workshop, insisted on anonymity as she was not comfortable raising the issue outside of a safe space. Men in the University are also aware of what it takes to speak up against unacceptable behaviour (see Allies in the classroom).

The relevant meta-themes also identify the importance of collaboration, language, respect; and reaching a point where everyone benefits - it is not just individual success that matters.

Related recipes and papers include: Recognise your privilege, The power of language: moving beyond past harms and present hurts, The current landscape at the University of Edinburgh, Say something, Damning with faint praise, Not just " $a$ ladies' problem", Tackling difficult situations - supporting your staff and students, Educated Pass: engaging young males from low socio-economic status backgrounds with learning, Support for students who report sexual harassment or assault.

\section{Insist upon and model open and honest awareness, reflection and action}

There is an absolute need for reflective practice.

All change starts with open and honest recognition and awareness, as so many of the recipes in this book demonstrate. Many of the recipes share their author's experience of developing a culture of open reflection and awareness for all members of staff and students.
Senior women leaders in Geosciences prefer to lead with democratic and pacesetting styles rather than in coercive or authoritarian ways (see Leadership styles and approaches in GeoSciences) and senior leaders, discussing honest reflection and action (see Leadership perspectives on gender equality) acknowledged that creating an inclusive culture takes effort and courage.

A recent example of honest awareness from a UK university about equality issues comes from Imperial College which, acting on concern about sexism within university sports, commissioned and published some of the results from an independent research project about gender and culture within its organisation (Taylor, 2016). The report, which is published on the university's website, documents negative views of staff and students including the perception held by some that misogyny is "ingrained" at the institution. In an article in the Independent newspaper, the researcher Dr Phipps noted that: "Imperial College has shown tremendous courage in not just ticking the boxes, but appointing a feminist team to do in-depth research on its institutional culture. We feel confident that positive changes will take place because of our study." The Provost of Imperial commented that "we are committed to ensuring gender equality and eradicating sexist behaviour wherever we can, at all levels. These findings remind us that we cannot stand still. We must do better." This demonstrates awareness and reflection on the problems within a culture, which can be the first step to cultural change.

Having the tools to do this proactively and positively is important. Academic debate and workplace dialogue are not necessarily congruent and some types of academic discourse are just not suitable in normal working situations, for example argument based on authority of methods, models or even people; and on the assumption that there is some correct answer or way of doing something. Similarly, in those subjects where more complex answers exist, the arguments drag on and the 
literature expands to fill entire libraries. In equitable workplaces, dialogue, negotiation, collaboration and what Charles Handy calls 'decent doubt' should be the norm. Like any other academic process, applying an appropriate methodology as well as specific methods is vital!

Recipes and papers abound to support this recommendation. Here are just some of them: Unravelling rhetoric, Grumpy, Say something, Be vigilant with your vocabulary, Challenging bias, Pause, Leadership styles and approaches in GeoSciences, Leadership perspectives on gender equality.

\section{Know, develop and celebrate yourself}

There is a drip, drip, drip of undermining comments you face as a female academic. It just becomes normal.

Authors - students and staff at all stages of their studies and their careers - wrote about the difficulty of developing and maintaining a healthy level of confidence and self-esteem in the face of the "drip, drip, drip" of an historically androcentric (and predominantly white, middle-class) context. See, for example, undermining comments that aren't even meant as such (see Damning with faint praise) and whose speaker would be - and is - horrified when confronted about them; and the choice between being perceived as competent or likeable (see Likeability and the double bind). There are many recipes where the author (often anonymously) tells us how she (and sometimes he) has encountered these societal pressures and dealt with them.

One of the most compelling personal accounts is Theresia Mina's story about how she - determinedly, assertively and respectfully - convinced her family to let her study. In doing so, she made her excellent academic record and letters from mentors part of the argument about why her academic future was worth investment. Theresia knew her own potential even when those closest to her did not yet recognise it. (See How to convince your family to let you study.)

The recipes recount personal successes, and the satisfaction of seeing how many small triumphs of courage, wit and humour can help a whole culture begin to shift. Other recipes look at the opportunities there are for professional development in parallel with the personal journey; often the two are closely interlinked - learning how to talk about your achievements without feeling either an imposter or becoming a 'humblebragger' takes courage as well. Making a choice to step outside of a research career, or to plan a career consciously and carefully rather than just falling into the next thing that presents itself, takes skill and support.

The titles speak for themselves: Dealing with imposter syndrome, Becoming visible in meetings, Raising your profile within your organisation, Career coaching for individuals, Stereotype threat, Talking about your achievements, Likeability and the double bind, Research isn't the only route, Career progression on a shoestring, Planning your career, Defining excellence, How to convince your family to let you study.

\section{Remove bias (conscious and unconscious) in learning and teaching}

There are issues with gender in the curriculum. Who teaches what, how teaching is perceived by students, what is taught.

The authors are very aware that teaching practice, as well as curriculum content, influences young minds. How we approach pedagogy, opportunities, ideas, support, pastoral care, tolerance, attitudes, are all reflected in our teaching practice - both explicitly and implicitly. There are recipes written by students who have dealt with challenging classroom situations, and also by members of the academic staff who have faced similarly difficult 
circumstances. Curriculum content is flagged up by contributors, students as well as staff, with the challenge to consider whether the topics discussed in classrooms include the intellectual contributions of women and give due weight to issues affecting women in society.

In addition to attending to bias in curricular content across disciplines, serious attention should be paid to the study of gender equality itself. Although there are academic staff whose research areas (English, French, German, Film, Russian, Hispanic Studies, Education and others) encompass gender and feminism, there is no department of Gender Studies at the University of Edinburgh. In 2014 an Edinburgh University Students' Association (EUSA) 'academic campaign' to create a Gender Studies department at the University convened a working group of academics with the vision of creating a Gender Studies course which would be available to all students.

There is now a Masters by Research degree in Gender and Culture, based in the School of Social and Political Science. There is ongoing work with EUSA to extend the subject and make it available to all students. As we observed earlier, how values are implemented in practice really matters; and in an academic institution value is demonstrated by the attention paid to it $-a$ degree is the ultimate value for students. Raising the status of gender as a subject of study at the University is a positive step.

The ideal of the University as a place of learning and teaching relies on an appropriate culture of self- and peerreflection and critique. Being aware of the necessity of creating and maintaining a culture of support is vital. Here are a few of the recipes that do this: Creating a safe space for classroom discussions, Allies in the classroom, Grow and succeed with fellow students, Gender balancing the curriculum, Show your daughters the joys of science, How to convince your family to let you study.

\section{Continuously balancing gender is the outcome}

It can be hard to challenge behaviours that are so embedded that you stop noticing. Small every day injustices... but tackling it is not only good for women but good for everyone.

It is the process of enabling equity that matters. The process of making changes, changes the people involved (see Finding my voice). As editors we have each responded to the challenges of the project by rethinking our ideas and preconceptions and often by learning new behaviours.

We have continuously thought that we had reached a final point, an outcome, in our exploration, only to find that there is always more to do. Closing down the content-gathering was painful! There are always other groups of people who would benefit from more equitable chances in higher education.

All of the recipes, case studies and evidence in this book, share a common idea - change can happen and it starts with identifying something, acting on it and then monitoring the changes. The recipes that describe their process, naturally extend that last step to checking that the intended effect was achieved as well as any potential unintended effects (see 2. Take a pragmatic, evidence based approach above).

They also all recognise that these matters are inherently social, emergent and complex. There are rarely any single solutions to any of the issues around gender equity in academia. They require a process of 'making equitable' to also be collectively engaging, continuous and nuanced. Reframing gender equity as a process, not just a set of policies and procedures or single activities, ensures that the balance between collective and individual responsibility can be developed and maintained. That is, each person has a responsibility within the culture to contribute to the process of equity 
in gender. Similarly, the institution or organisation itself must actively support such processes, and in no way impede or discourage the people engaged in them.

Emily Yarrow's article Gender and the Research Excellence Framework (REF) reminds us of what can happen when new inclusive processes are forgotten in a drive to meet governmental pressures. The high stakes of the REF apparently led to bias in selection of academics for inclusion in the REF itself, and biased REF-related hiring and salary decisions will have a lasting impact on equality across the UK higher education sector.

It is possible that processes designed through Athena SWAN action groups were not sufficiently well embedded within universities to withstand the fiercely competitive game of optimising REF returns. However, it shouldn't be that departments tick the boxes to get their Athena SWAN report submitted and then move on to sorting out their REF profile; departments should habitually use biasreduction strategies to maintain gender equality (see A model for change).

The meta-themes are all relevant here: responsibility, reflection, challenging bias, language, well-being, humour, personal skills and principles and - of course - processes. Recipes and papers here include: The power of language, Raising your profile within your organisation, Gender and the Research Excellence Framework, Finding my voice.

\section{Create an environment that enables people to flourish}

You need an environment where people feel safe to challenge inappropriate behaviour. You need trust. People need to feel they can talk about it and be listened to.

'Environment' encompasses social and physical spaces, people, resources and processes: they are deeply interlinked. The gendered nature of buildings (see Asking for equitable buildings) explores how our built environment is an operational as well as a fixed entity that meets some human need. Bringing the affordances (things that hold the possibility of an action) into alignment with a positive organisational culture is as important as the claimed values and practices. The University has policies intended to support physiological issues, and authors' recipes examine what happens when the physical and financial assets to support policy are provided generously (for example the Roslin Insitute's childcare provision).

The contributors also tell us about how protracted policy implementation affects them. The move toward genderneutral toilets, for example, is slow. The impact of this can be read plainly in the Unapologetically Me piece (see Perspectives from students) where a student has to decide each morning whether they look feminine enough to get into the women's toilets without getting chucked out, a point reinforced by a student's illustration of a toilet gender guard. Student parents are also arguing for environmental changes to accommodate their needs.

The physical environment can also have a strong emotional impact, for example, crucial interviews, meetings and examinations held in rooms where every portrait on the walls is male (see You can't be what you can't see) something that male visitors to the room don't notice and aren't affected by, but which triggers stereotype threat and imposter syndrome (see Dealing with imposter syndrome) in women; and the impact, reported by female students, that a very masculine gym environment can have on them (see Reflections on exercise and sport in the University).

Perhaps unsurprisingly, the learning and teaching spaces we create reflect the complex socio-political spaces the academy inhabits. The way a room is 
laid out can enhance or inhibit learning, especially collaborative practice; more than one of the editorial team is in the habit of arriving early in any seminar room to rearrange the furniture.

Recipes include: Rose surprise - when your period comes early, You can't be what you can't see: visible celebration of notable women, Reflections on exercise and sport in the University, Exercise and sport for all, What have you done to my squash courts!, Asking for equitable buildings, Creating a safe space for classroom discussions.

\section{Recognise and honour the greater context}

We need a different mindset which is more relevant to flexible careers $-[. .$.$] what happens outside$ work, what happens in work.

The academy exists in, and is part of, society. Like any other employment space, the relationships between work, lifestyle, family, and society generally can be difficult.

Many of the recipes in this book discuss the difficulties of balancing academia with family commitments. That balancing should even be required shows that we have a long way to go before achieving any dream of socially sustainable employment. But at the very least, universities must take on board "Close the gap between policy and practice" and ensure that opportunities such as flexible working or part-time working are supported fully. Very often the implementation of these falls far short of the strategic aims or values set out. Staff deal with these shortfalls on a daily basis with significantly raised stress levels and high workloads; surveys of the academic workforce document lower than average well-being across a range of indicators (see Gender in higher education: the current landscape in the UK).

Hierarchical perception of disciplines and careers can be pernicious (as a pure physics student was heard to say: "Art students are top of the heap, then pure maths and pure physics, then it's downhill from there"). Traditional linear career paths are not necessarily 'better', and 'leaky pipeline' discussions risk narrowing the focus and ignoring alternative approaches. Nonlinear and alternative career directions already exist in academia and in other employment domains. The University of Edinburgh is beginning to support and develop such options, and some of the recipes recognise and celebrate this. Success is being redefined. Recipes include: Raising your profile within your organisation, Career coaching for individuals, Advertise all opportunities large and small, The kids are alright, Flexible working: being realistic, Deadlines and diapers: being an academic dad, Career progression on a shoestring, Proactive promotion.

\section{Be a beacon}

We know that the influence the academy has on knowledge, culture, policy, society, economics, etc. is significant. The minds being shaped in and by the academy will go on to shape and create society in turn. Similarly, the direct influence of the academy in policy and legislation is also significant. At these levels it is even more important to ensure that the principle of "closing the gap between policy and practice" is embedded in the culture. If the external actions of a university do not match their stated values, this is noticed by staff and students.

Part of achieving an Athena SWAN Gold award at institutional level is to be a beacon for gender equality, and to take responsibility for helping other universities to improve. Being a beacon also means more than aspiring to have slightly higher proportions of women students than the UK average for a specific discipline, particularly if the national average is in single digits. We can propose and achieve higher targets for the proportion of women who are included in the more senior levels 
of academia, as illustrated in the recipe about gender balancing seminar speakers in which the initial target of $30 \%$ women presenters was comfortably surpassed.

At the 'domestic' level, universities can influence neighbours in the academic ecology, such as academic publishing processes and publishers; and neighbours in the ecology of work practice, including quality approaches and standards agencies; policy advice and legislation representation; knowledge transfer and business interrelations. And the universities' influence on arts and culture, employment and work/life issues is also considerable.

Universities can support socio-political influence in an open way, recognising that the value of the academy is in its contribution to the society from/ within which it exists. The methods by which this is achieved are also open to creativity - how the University represents and embodies divergent views is of significant value to wider society.

The more difficult consideration is the incongruence of inaction - where Universities choose to make no responses, which may seem to contradict their stated values. The tensions here are clear, and the incongruence between stated values and actual practice can be damaging; but beyond any simple conceptualisation of these as power negotiations there is yet another way of looking at this. Universities are significant places of novelty and change and they cannot help but contribute to that change - positively or otherwise. The academy can reach out and be proud of this, taking a lead and demonstrating leadership rather than simply driving inevitable change.
The academy is a place within which the future is imagined and then created. When this becomes an equitably shared imagining, the future, too, becomes an equitably shared one. Margaret Mead wrote:

$$
\begin{aligned}
& \text { Never doubt that a small } \\
& \text { group of thoughtful, } \\
& \text { committed citizens can } \\
& \text { change the world; indeed, it's } \\
& \text { the only thing that ever has. }
\end{aligned}
$$

\author{
(http://www.interculturalstudies. \\ org/faq.html)
}

While, as an editorial team, we would not claim to change the world, as a small group of thoughtful and committed citizens, we and all our contributors do not doubt that the writing of, illustrating, reading, reflecting on, and acting upon this book is part of creating an equitable academic world.

That is a most inspiring outcome. 